\title{
The solution of Navier Stokes equation for MHD Casson fluid using DTM-Pade Approximation
}

\author{
H. V. Gangamani, Nivedita
}

\begin{abstract}
The flow problem of Magnetohydrodynamic three-dimensional Casson fluid past a porous linearly stretching sheet is investigated analytically. The flow governing equations are reduced to a set of nonlinear ordinary differential equations using the similarity transformations and solved using an efficient and suitable mathematical technique, the differential transform method (DTM), in the form of convergent series, by applying Pade approximation and the results are validated using Runge-Kutta-Fehlberg Numerical Method. DTM-Pade proves to be an efficient method in solving the Non-linear Differential Equations. By increasing the non-Newtonian fluid parameter the fluid reduces to the Newtonian Fluid is illustrated using graphs. It is also clear that the Casson fluid parameter, stretching parameter, Hartmann number and porosity parameter increase with increase in the velocity profiles of the fluid.
\end{abstract}

Index Terms - MHD Casson Fluid, Boundary Layer, Rotating Disc, DTM-Pade, Approximation, Porous medium.

\section{INTRODUCTION}

Fluid mechanics has a wide range of applications including mechanical engineering, biomedical engineering, geophysics, astrophysics and biology [11]. Researchers have shown enormous interest in three-dimensional flow of non-Newtonian fluid due to its extensive practical importance in industry and engineering[16]. Nonlinear phenomena have important effects on applied Mathematical problems. The importance of obtaining the exact or approximate solutions of nonlinear partial differential equations (NLPDEs) in applied Mathematical problem is the most formidable problem that needs various methods for exact or approximate solutions. Most of nonlinear equations do not have a precise analytic solution; so numerical methods have largely been used to handle these equations.

Some analytic techniques for nonlinear equations which are classic analytic methods such as Lyapunov method, perturbation techniques, Homotopy perturbation method (HPM), Homotopy analysis method (HAM) and the Differential Transform Method.

Non-Newtonian fluids have received more attention and significance than Newtonian fluid in the study of flow of viscoelastic fluids[11] was first presented by Casson in 1995 . This model is cast off by fuel engineers in the understanding of adhesive slurry and is improved for forecasting high

Gangamani H V, Post Graduate Department of Mathematics, Bengaluru Central University/ MES College/ Mysore Education Society, Bangalore, India, 9148325125

Nivedita, Post Graduate Department of Mathematics Bengaluru Central University / MES College / Mysore Education Society, Bangalore, India sheer-rate viscosities when only low and traditional sheer-rate data are accessible. Boundary layer flow over stretching plate was first presented by Crane.

A fluid that does not follow Newton's law of viscosity is called Non-Newtonian fluid. The most popular among these fluids is the Casson fluid. Casson fluid is defined as shear thinning liquid which is assumed to have an infinite viscosity at zero rate of shear, a yield stress below which no flow occurs and a zero viscosity at an infinite rate of shear [6].

Most Non-Newtonian fluids, Casson fluid and nanofluid are modeled by non-linear differential equations. Most effective methods for solving non-linear problems is the DTM, first proposed by Zhou[9]. DTM has been applied to many problems such as linear partial differential equations of fractional order, non-linear oscillating system, multiorder fractional differential equations, hyper-chaotic Rossler system etc. DTM constructs, for differential equations an analytical solution in the form of power series but these power series is not useful for large value of $\eta$, say $\eta \rightarrow 0$.

Therefore the combination of the series solution through the DTM or any other series solution method with the Pade approximation. DTM-Pade has been successfully employed to solve many types of non-linear problem such as MHD flow in a laminar liquid film and nano boundary layers over stretching surfaces[7].

The non-linear equations of the flow field has been solved by Differential transform method empowered by Pade approximants analytically and Runge-Kutta-Fehlberg[12] method with shooting techniques is used to solve Navier stokes' equation numerically in this paper.

\section{FLOW DUE TO A ROTATING DISC OF INFINITE RADIUS}

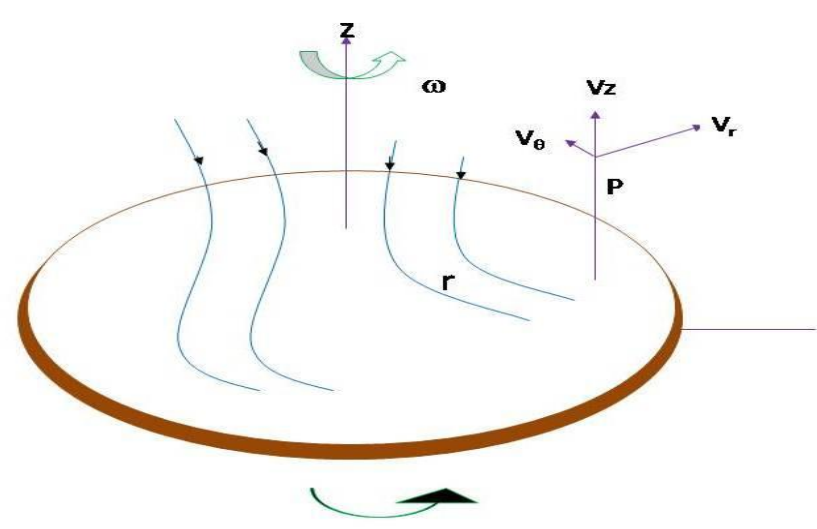

Fig.1: Schematic of Flow due to a rotating disc

Consider the flow due to a rotating disc which rotates with an angular velocity $\omega$ about an axis perpendicular to its plane. The disc is considered to be of infinite radius. Due to the centrifugal forces the fluid near the disc will be thrown 
outward and it is compensated by flow in the axial direction towards the disc[5]. Thus all the three components of velocity in the cylindrical polar coordinates will exist. Accordingly the governing equations are in the form

$\frac{1}{r} \frac{\partial}{\partial r}\left(r v_{r}\right)+\frac{1}{r} \frac{\partial v_{\theta}}{\partial \theta}+\frac{\partial v_{z}}{\partial z}=0$

$\rho\left(\frac{D v_{r}}{D t}-v_{\theta}{ }^{2}\right)=\rho f_{r}-\frac{\partial p}{\partial r}+\mu\left(\nabla^{2} v_{r}-\frac{v_{r}}{r^{2}}-\frac{2}{r^{2}} \frac{\partial v_{\theta}}{\partial r}\right)$

$\rho\left(\frac{D v_{\theta}}{D t}-\frac{v_{\theta} v_{r}}{r}\right)=\rho f_{\theta}-\frac{\partial p}{\partial \theta}+\mu\left(\nabla^{2} v_{\theta}-\frac{v_{\theta}}{r^{2}}+\frac{2}{r^{2}} \frac{\partial v_{r}}{\partial \theta}\right)$

$\rho \frac{D v_{z}}{D t}=\rho f_{z}-\frac{\partial p}{\partial z}+\mu \nabla^{2} v_{z}$

Subsequent boundary conditions are

$$
\begin{gathered}
z=0: v_{r}=0, \quad v_{\theta}=r \omega, \quad v_{z}=0 \\
z=\infty: v_{r}=0, \quad v_{\theta}=0
\end{gathered}
$$

In the absence of body forces, we will consider the following possibilities for the velocity and pressure distribution

$$
\begin{aligned}
& v_{r}=\omega r f(z), \quad v_{\theta}=\omega r g(z), \quad v_{z}=(v \omega)^{\frac{1}{2}} h(z), \\
& p=\rho v \omega p(z)
\end{aligned}
$$

substituting (2.6) in (2.1) - (2.4) under the absence of external force, we obtain

$$
\begin{aligned}
& 2 f+\left(\frac{v}{\omega}\right)^{\frac{1}{2}} h^{\prime}=0 \\
& f^{2}-g^{2}+\left(\frac{v}{\omega}\right)^{\frac{1}{2}} f^{\prime} h=\left(\frac{v}{\omega}\right) f^{\prime \prime} \\
& 2 f g+\left(\frac{v}{\omega}\right)^{\frac{1}{2}} g^{\prime} h=\left(\frac{v}{\omega}\right) g^{\prime \prime} \\
& h h^{\prime}=-p+\left(\frac{v}{\omega}\right)^{\frac{1}{2}} h^{\prime \prime}
\end{aligned}
$$

where prime denotes the differentiation with respect to $z$. To remove the constant coefficients $(v / \omega)^{\frac{1}{2}}$, we will use the following transformations.

$$
\begin{aligned}
& \eta=(v / \omega)^{\frac{1}{2}} z, f(z)=F(\eta), g(z)=G(\eta), \\
& h(z)=H(\eta), p(z)=P(\eta)
\end{aligned}
$$

Using equation (2.8) in equations (2.7), we arrive to equations in the form

$$
\begin{aligned}
& 2 F+H^{\prime}=0 \\
& F^{2}-G^{2}+F^{\prime} H=F^{\prime \prime} \\
& 2 F G+G^{\prime} H=G^{\prime \prime} \\
& H H^{\prime}=-P+H^{\prime \prime}
\end{aligned}
$$

The transformed boundary conditions are,

$$
\begin{aligned}
& f(0)=0, \quad g(0)=1, \quad h(0)=0 ; \quad f(1)=a_{1}, \\
& g(1)=b_{1} \quad \text { where } a_{1}=0.510, \quad b_{1}=-0.616
\end{aligned}
$$

Using the appropriate DTM transformations in the set of equations (2.9), we have,

$2 f(k)+(k+l) H(k+l)=0$

$\sum_{l=0}^{k} F(l) F(k-l)-\sum_{l=0}^{k} G(l) G(k-l)+$

$\sum_{l=0}^{k}(k-l+1) F(k-l+1) H(l)=(k+1)(k+2) F(k+2)$

$2 \sum_{l=0}^{k} F(l) G(k-l)+$

$\sum_{l=0}^{k}(k-l+1) G(k-l+1) H(l)=(k+1)(k+2) G(k+2)$

with boundary conditions:

$F(0)=0, G(0)=1, H(0)=0 ; F(1)=a_{1}, F(1)=b_{1}$

The solutions in the form of expansion for small $\eta$ is given by

$$
\begin{aligned}
& F=a_{1} \eta-\frac{1}{2} \eta^{2}-\frac{1}{3} b_{1} \eta^{3}-\frac{1}{12} b_{1}^{2} \eta^{4}-\ldots \ldots \\
& G=1+b_{1} \eta+\frac{1}{3} a_{1} \eta^{3}-\frac{1}{12}\left(a_{1} b_{1}-1\right) \eta^{4}-\ldots \ldots \\
& H=-a_{1} \eta^{2}+\frac{1}{3} \eta^{3}+\frac{1}{6} b_{1} \eta^{4}+\ldots \ldots
\end{aligned}
$$

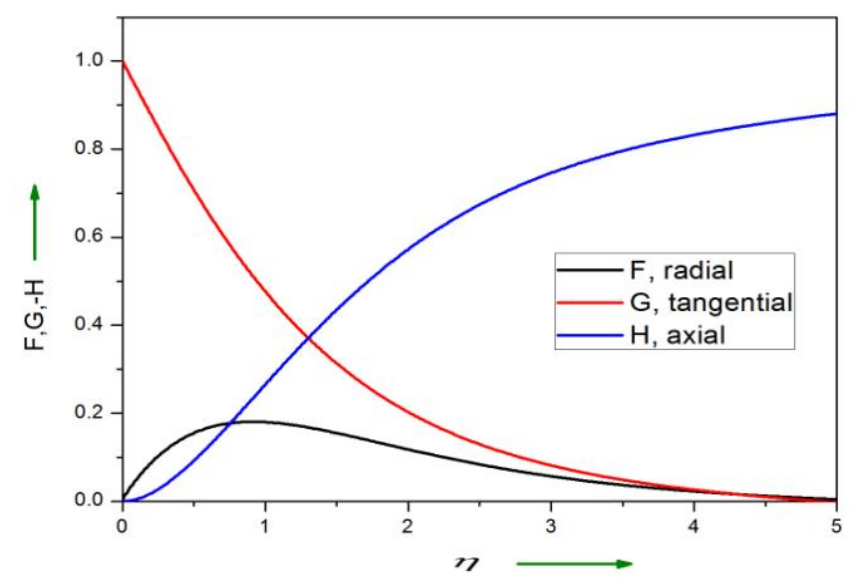

Fig. 2: The velocity distribution near a rotating disc for an infinite radius.

It can be observed from the figure that $\mathrm{F}, \mathrm{G}$ and $\mathrm{H}$ tend to their limiting value asymptotically but for practical purpose $\eta=5$ and the corresponding values of $\mathrm{z}$, which we call as $\delta$ from the equation(2.8)

$$
\delta=5(v / \omega)^{\frac{1}{2}}
$$


if $v / \omega$ is small then $\delta$ will be small which gives rise to a boundary layer type of flow which is precisely explained by the model in the following section.

\section{MHD CASSON FLUID PAST A POROUS LINEARLY STRETCHING SHEET}

\section{A Mathematical Formulation}

This model is used by fuel engineers. Most of the Casson fluids are modeled by nonlinear partial differential equations. Consider a three-dimensional (3D) incompressible flow past a stretching sheet. It is assumed that the sheet stretches along $\mathrm{xy}$-plane, while the fluid is placed along z-axis.

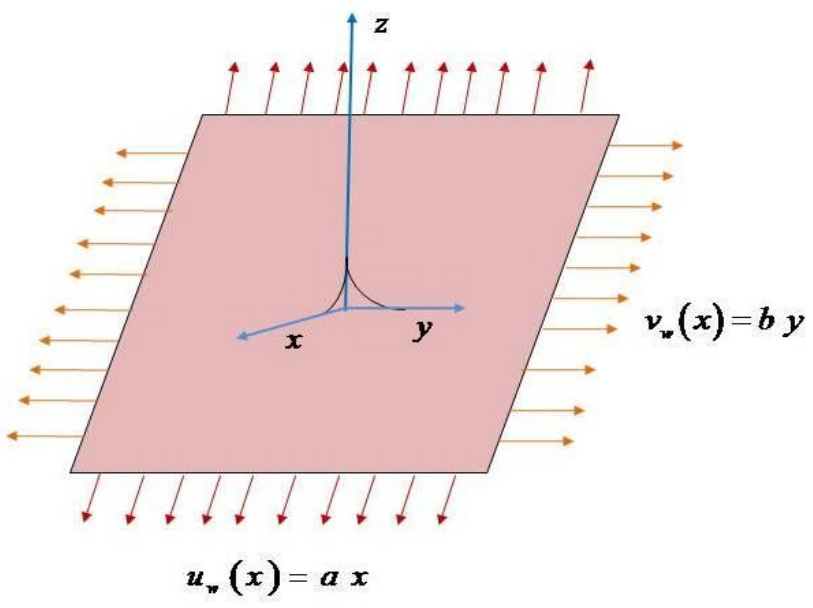

Fig.3: Nanofluid stretching sheet flow physical model

The boundary layer equations of 3-D incompressible Casson fluids are,

$$
\begin{aligned}
& \frac{\partial u}{\partial x}+\frac{\partial v}{\partial y}+\frac{\partial w}{\partial z}=0 \\
& u \frac{\partial u}{\partial x}+v \frac{\partial u}{\partial y}+w \frac{\partial u}{\partial z}=v\left(1+\frac{1}{\beta}\right) \frac{\partial^{2} u}{\partial z^{2}}-\frac{\sigma B^{2}}{\rho f} u-\frac{v}{k} u \\
& u \frac{\partial v}{\partial x}+v \frac{\partial v}{\partial y}+w \frac{\partial v}{\partial z}=v\left(1+\frac{1}{\beta}\right) \frac{\partial^{2} v}{\partial z^{2}}-\frac{\sigma B^{2}}{\rho f} v-\frac{v}{k} v
\end{aligned}
$$

Where, $\mathrm{u}, \mathrm{v} \mathrm{w}$ denote the velocities in $\mathrm{x}, \mathrm{y}$ and $\mathrm{z}$ directions respectively, $\beta=$ Casson fluid parameter, $v=$ Kinematic viscosity, $\mathrm{B}=$ magnetic induction, $\mathrm{K}=$ porous medium permeability

Boundary conditions are,

$$
\left.\left.\begin{array}{l}
u=a x=u_{w}(x) \\
v=b y=v_{w}(y)
\end{array}\right\} \text { at } \quad z=0 \quad \begin{array}{l}
u \rightarrow 0 \\
v \rightarrow 0
\end{array}\right\} \text { at } \quad z \rightarrow \infty
$$

where $\mathrm{a}$ and $\mathrm{b}$ are positive constants, $u_{w}$ and $v_{w}$ are stretching velocities in $\mathrm{x}$ and $\mathrm{y}$-direction respectively. Introducing the similarity transformations $u=a x f^{\prime}(\eta), v=b y g^{\prime}(\eta)$

$w=-\sqrt{a v}[f(\eta)+c g(\eta)]$

$\eta=\sqrt{\frac{a}{v} z} \quad$ Where, $c=\frac{b}{a}$

using the similarity transformation in equation (3.5) in equations (3.1) - (3.3) we have coupled non-linear differential equations

$\left(1+\frac{1}{\beta}\right) f^{\prime \prime \prime}+(f+g) f^{\prime \prime}-\left(f^{\prime}\right)^{2}-M^{2} f^{\prime}-\lambda f^{\prime}=0$
$\left(1+\frac{1}{\beta}\right) g^{\prime \prime \prime}+(f+g) g^{\prime \prime}-\left(g^{\prime}\right)^{2}-M^{2} g^{\prime}-\lambda g^{\prime}=0$

\section{B Analytical approximation by means of the DTM-Pade}

using the suitable DTM transformation $\mathrm{F}(\mathrm{k})$ and $\mathrm{G}(\mathrm{k})$ are transformed equations of $\mathrm{f}(\mathrm{k})$ and $\mathrm{g}(\mathrm{k})$ which are given by,

$$
\begin{aligned}
& f(\eta)=\sum_{k=0}^{\infty} F(k) \eta^{k} \\
& g(\eta)=\sum_{k=0}^{\infty} G(k) \eta^{k} \\
& \left(1+\frac{1}{\beta}\right)(k+1)(k+2)(k+3) F(k+3)= \\
& \sum_{r=0}^{k}(r+1) F(r+1)(k-r+1) F(k-r+1) \\
& -\sum_{r=0}^{k}(k-r+1)(k-r+2) F(r) F(k-r+2) \\
& -\sum_{r=0}^{k}(k-r+1)(k-r+2) G(r) F(k-r+2) \\
& +\left(M^{2}+\lambda\right)(k+1) F(k+1) \\
& \left(1+\frac{1}{\beta}\right)(k+1)(k+2)(k+3) G(k+3)= \\
& \sum_{r=0}^{k}(r+1) G(r+1)(k-r+1) G(k-r+1) \\
& -\sum_{r=0}^{k}(k-r+1)(k-r+2) F(r) G(k-r+2) \\
& -\sum_{r=0}^{k}(k-r+1)(k-r+2) G(r) G(k-r+2) \\
& +\left(M^{2}+\lambda\right)(k+1) G(k+1)
\end{aligned}
$$

And the corresponding boundary conditions are 
$F(0)=0, \quad F(1)=1, \quad F(2)=a$,

$G(0)=0, \quad G(1)=0.5, \quad F(2)=b$

Using equation (3.11) in equations (3.9) and (3.10) and the substituting in equation (3.8), we obtain the power series solution in the following form

$$
\begin{aligned}
& f(\eta) \approx \eta+a \eta^{2}+\frac{\left(1+\lambda+M^{2}\right)}{6\left(1+\frac{1}{\beta}\right)} \eta^{3} \\
& +\frac{2 a\left(\lambda+M^{2}\right)+a}{24\left(1+\frac{1}{\beta}\right)} \eta^{4}+\ldots \ldots \\
& g(\eta) \approx 0.5 \eta+b \eta^{2}+\frac{0.5\left(0.5+\lambda+M^{2}\right)}{6\left(1+\frac{1}{\beta}\right)} \eta^{3} \\
& +\frac{2 b\left(\lambda+M^{2}\right)}{24\left(1+\frac{1}{\beta}\right)} \eta^{4}+\ldots \ldots
\end{aligned}
$$

\section{RESULTS AND DISCUSIIONS}

We have plotted the velocity distribution near a rotating disc in graph in Figure 2 and we can see from the graph that F, G,H tend to their limiting values asymptotically using which the boundary layer type can be estimated for a rotating fluid.
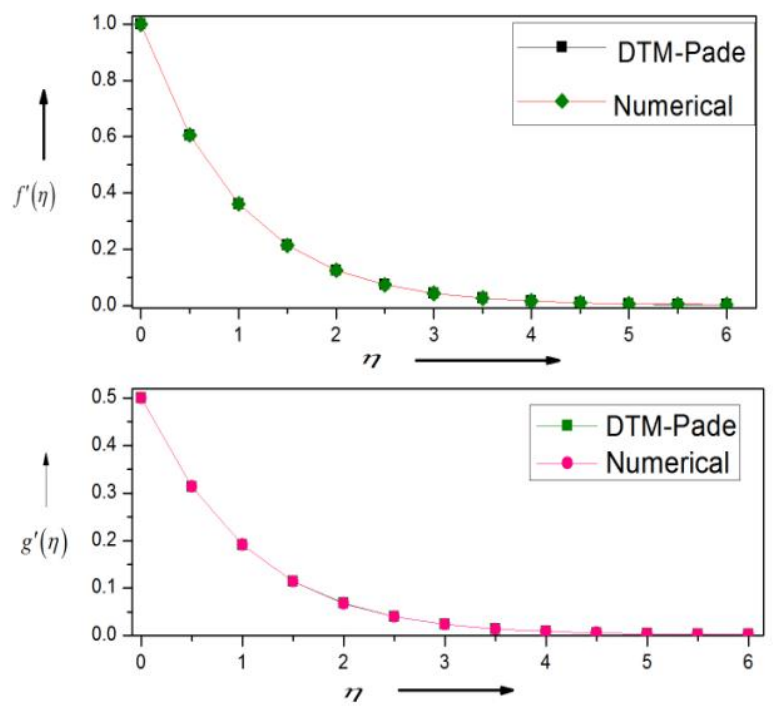

Fig. 4: Velocity profiles of $f^{\prime}(\eta)$ and $g^{\prime}(\eta)$ using DTM-Pade and Runge-Kutta-Felhberg Method.

The graph in figure 4 shows that DTM-Pade and Numerical results are in very good agreement for the velocity profiles $f^{\prime}(\eta)$ and $g^{\prime}(\eta)$ using Casson fluid parameter $\lambda=M=c=0.5, \beta=1$. The graphs in Figures 5 and 6 gives the velocity profiles for the Casson fluid parameter and the porosity Parameter. It shows that effects of increasing the non-Newtonian parameter fluid parameter $\beta$ reduces the fluid to be more Newtonian in nature and when increasing the values of the porosity parameter $\lambda$, within the boundary layer.
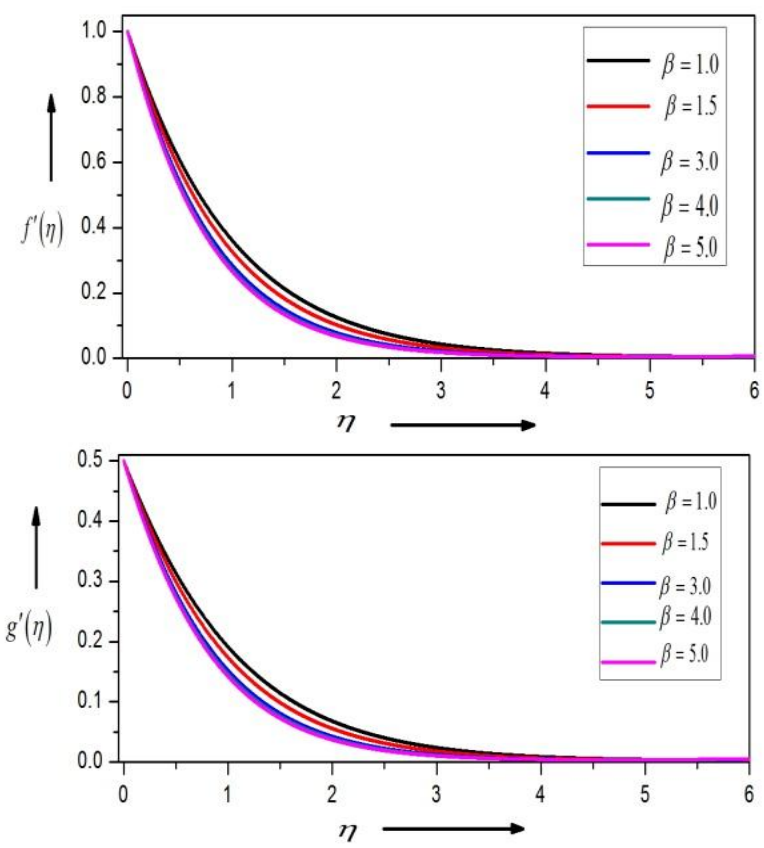

Fig. 5: Velocity profiles $f^{\prime}(\eta)$ and $g^{\prime}(\eta)$ with varying $\beta$
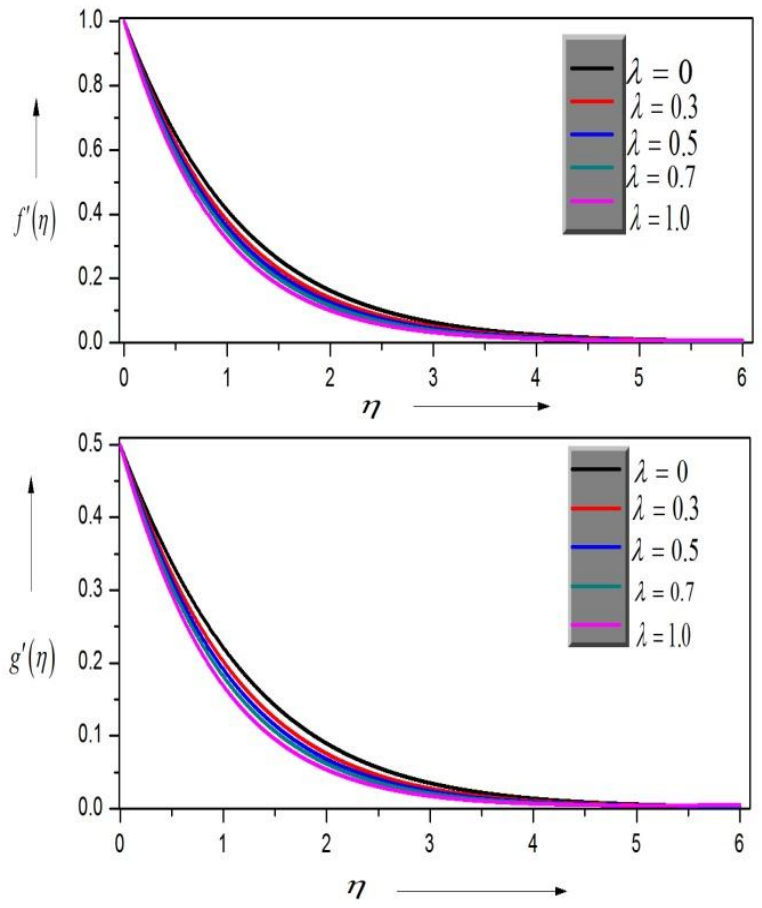

Fig.6: $f^{\prime}(\eta)$ and $g^{\prime}(\eta)$ with varying Porosity

$$
\operatorname{Parameter}(\lambda) \text {. }
$$

Also for higher values of $\lambda$, the thickness boundary layer decreases and also there is a reduction in the boundary layer 
thickness. The graphs in figure7 shows the effects if increasing Hartmann Parameter, we see that the magnitude of the velocity reduce when the values of $M$ increase. The graphs in figure8 depict that the velocity $f^{\prime}(\eta)$ reduce at increased values of the stretching parameter $\mathrm{c}$, while $g^{\prime}(\eta)$ varies with respect to various values of the stretching parameter c.
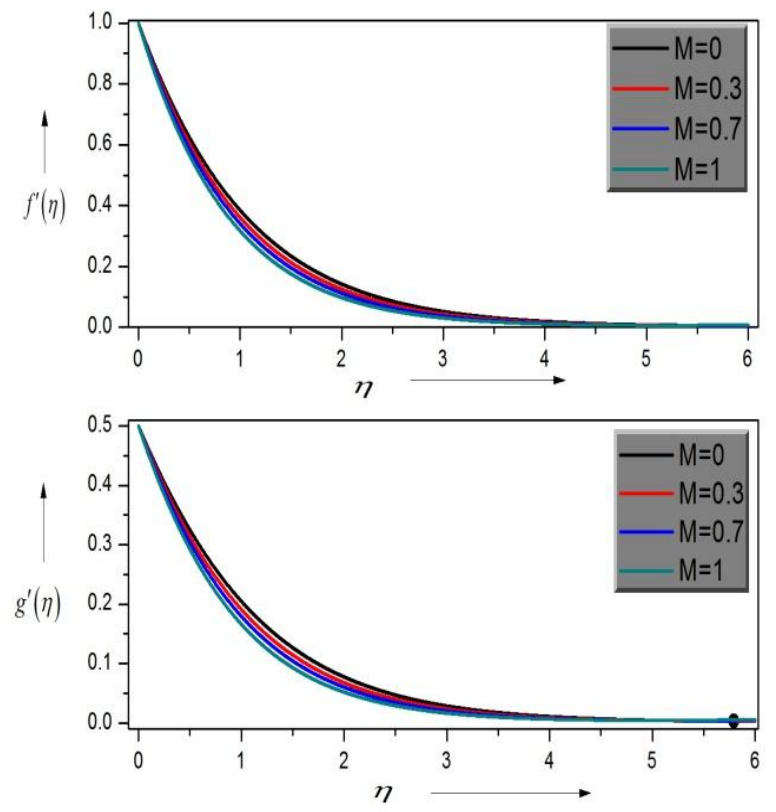

Fig.7: $f^{\prime}(\eta)$ and $g^{\prime}(\eta)$ with varying Hartmann Number(M).
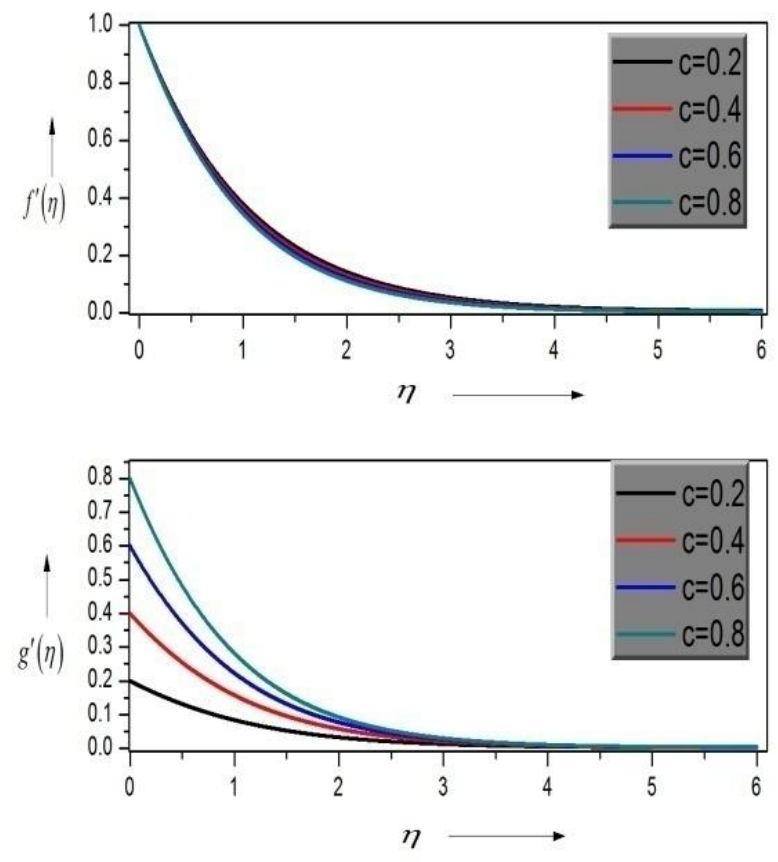

Fig. 8: $f^{\prime}(\eta)$ and $g^{\prime}(\eta)$ with varying Stretching

\section{Parameter( c).}

\section{CONCLUSION}

The solution that we have obtained for the coupled non-linear equations are consistent with the numerical results. These results are obtained without requiring linearization, discretization and perturbation. DTM-Pade proves to be an efficient method in solving the non-linear equations occurring in different engineering and applied fields.

\section{ACKNOWLEDGMENT}

The study is supported by VGST GRD 105 Government of Karnataka.

\section{REFERENCES}

[1] H. Liu and Y. Song, Differential transform method applied to high index differential algebraic equations, Applied Mathematics and Computation, vol. 184, no. 2, pp. 748753, 2007.

[2] Supriya Mukherjee and Banamali Roy,"Solution of Reccati Equation with Variable Co- efficient by Differential Transform method International Journal of Nonlinear Science", 1749-3897,2012.

[3] Reza Hosseini, Sadegh Poozesh, and Saeed Dinarvand," MHD Flow of an Incompressible Viscous Fluid through Convergent or Divergent Channels in Presence of a High Magnetic Field, Journal of Applied Mathematics", V2012, ,available at doi:10.1155/2012/157067, 2012.

[4] M. M. Bhatti, M. M. Rashidi, Numerical simulation of entropy generation on MHD nanofluid towards a stagnation point flow over a stretching surface, Int. J. Appl. Comput. Math., 2, 115, 1, 2016.

[5] J. L. Bansal, Viscous Fluid Dynamics, "Oxford and IBH Publishing Co. PVT.,LTD.”,New Delhi, Pg No-1-5,109-113. 2005.

[6] P. A. Davidson, Introduction to Magnetohydrodynamics, Cambridge University Press, 2017.

[7] S. Baag, M.R. Acharya, G.C. Dash, "MHD flow analysis using DTM-Pade' and numerical methods", Amer. J. Fluid Dyn. 4(1), 2014,available at http://doi: 10.5923/j.ajfd.20140401.02

[8] Nadeem Tariq and Sajjad Hussain Hussain, "Megnetohydrodynamic Flow of Casson Fliuds over a Moving Boundary Surface,J. Appl. Environ. Biol. Sci.”, 7(4)192-200, 2017.

[9] J.K. Zhou. "Differential transforms and its application in electrical circuits, Huazhong University Press", Wuhan, China,1986.

[10] Ambrish Kumar Tiwari, Fauzia Raza, Jahan Akhtar, Mathematical Model for Marangoni Convection MHD Flow of Carbon Nanotubes through a Porous Medium, IAETSD Journal for Advanced Research in Applied Sciences, volume 4, 2394-8442, 2017 available at: http://iaetsdjaras.org.

[11] Robert W. Fox, Alan T. Mc Donald, "Introduction to Fluid mechanics", John Wiley and Sons, Pg no 23-25, 1994

[12] R.L. Burden, J.D. Faires, Numerical analysis, Cengage Learning, 2011.

[13] M.J. Jang, C.L. Chen, and Y.C. Liu, On solving the initial value problems using the differential transformation method, Applied Mathematics and Computation,115, 145-160, 2000.

[14] H. Liu and Y. Song, Differential transform method applied to high index differential algebraic equations, Applied Mathematics and Computation, vol. 184, no. 2, pp.748-753, 2007.

[15] Hamed Shahmohamadi, Mahdi Mohammadpour, A Series Solution for Three-Dimensional Navier-Stokes Equations of Flow near an Infinite Rotating Disk, Cambridge University Press, Wolfson School of Mechanical and Manufacturing Engineering, Loughborough University, Loughborough, UK available at: http://www.scirp.org/journal/wjm, http://dx.doi.org/10.4236/wjm.2014.45014

[16] Shahmohamadi, H. and Rashidi, M.M. , Explicit Solutions for Steady Three-Dimensional Problem of Condensation Film on Inclined Rotating Disk. International Journal of Fluid Mechanics Research, 38, 353-365,2011. http://dx.doi.org/10.1615/InterJFluidMechRes.v38.i4.50

[17] J. Biazar, M. Eslami, Differential transform method for systems of Volterra integral equations of the second kind and comparison with homotopy perturbation method, Int. J. Phys. Sci. 6 1207-1212,2011.

[18] Arikoglu, I. Ozkol, Solution of boundary value problems for integro-differential equations by using differential transform method, Appl. Math. Comput. $168,1145-1158,2005$. 
Dr. H.V. Gangamani born in Bangalore, Karnataka, India on 23rd August 1975 obtained M. Sc. securing Fourth Rank and successive Ph. D degree in the year 2009 for her work on Reflection and Transmission of Internal Gravity waves in electrically conducting and non-conducting fluids from Bangalore University, Bangalore, India under the able guidance of Prof. M. Venkatachalappa, FNA, FNASc, FISET. She has also done her Post Doctoral studies from the Indian Institute of Science, Bangalore as RASS Research Associate in COAS, She has participated in 18 Conferences and presented papers in 15 National and International Conferences. Presently She is working as an Assistant Professor and guiding M. Sc. Students for Projects in M.E.S. College, Malleswaram, Bangalore-560003, Karnataka, India. The topics of research interest are Fluid Dynamics, Applied Mathematics and Atmospheric Science

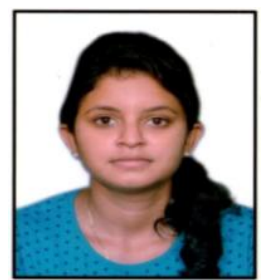

Nivedita, born in Orissa, India, M. Sc, Post graduate student of Bangalore University, having completed Bachelor of Science in integrated course from the regional institute of Education, Utkal university Orissa, has also successfully qualified in Central Teacher Eligibility test(CTET) and is open to new opportunities and eager to learn and improve skills to the best of her knowledge. She was also involved in the Project work during the course of her study in the Post graduate Department of Mathematics and Research Center in Applied Mathematics, entitled "An Analysis of Mathematical Modeling Using DTM-Pade Approximation For MHD Flows". She has also participated in workshops and conferences during her tenure in the Post Graduate Department of Mathematics. 